\title{
Research Article: Clinical Report \\ Prenatal identification of a novel Mutation in the MCPH1 Gene associated with autosomal recessive primary microcephaly (MCPH) using Next Generation Sequencing (NGS).
}

Ioannis Papoulidis ${ }^{1^{*}}$, Makarios Eleftheriades ${ }^{*}$, Emmanouil Manolakos ${ }^{1,3}$, Simoni Marina Liappi ${ }^{1}$, Anastasia Konstantinidou, Maria Papamichail5, Vassilios Papadopoulos ${ }^{6}$, Antonios Garas7, Sotirios Sotiriou ${ }^{8}$, Ioannis Papastefanou ${ }^{9}$, Georgios Daskalakis ${ }^{10}$, Aleksandar Ristic ${ }^{11}$

*Equal contribution / Shared co-first authorship

1. Access To Genome P.C., Clinical Laboratory Genetics, Athens-Thessaloniki, Greece. papoulidis@atg-labs.gr, liappi_sm@hotmail.com

2. Second Department of Obstetrics and Gynaecology, Aretaieion Hospital, Medical School, National and Kapodistrian University of Athens, Athens, Greece makarios@hotmail.co.uk.

3. Department of Medical Genetics, University of Cagliari, Binaghi Hospital, Cagliari, Italy. manolakos@atg-labs.gr

4. 1st Department of Pathology, School of Medicine, National and Kapodistrian University of Athens, Greece.

5. Postgraduate Programme "Maternal Fetal Medicine" Medical School National \& Kapodistrian University of Athens, Greece, mapapam@hotmail.com

6. Department of Obstetrics \& Gynecology, University of Patra, Greece. vpapado@upatras.gr

7. Department of Gynecology, Larissa Medical School, University of Thessaly, Larissa, Greece. garasant@yahoo.co.uk

8. Department of Clinical Embryology, Larissa Medical School, University of Thessaly, Larissa 41334, Greece. sotiriousoti@yahoo.gr

9. Fetal Medicine Clinic, Monis Petraki 4, Kolonaki, Athens, Greece,11521.jdpap2@yahoo.co.uk 
10.First Department of Obstetrics and Gynaecology, "Alexandra" Maternity Hospital, Medical School, National and Kapodistrian University of Athens, Athens , Greece. gdaskalakis@yahoo.com

11.Obstetric and Gynecological Clinic Narodni Front, Belgrade, Serbia galamedica@gmail.com

Short title: Papoulidis et al. PRENATAL IDENTIFICATION OF A NOVEL MUTATION IN THE MCPH1 GENE

\section{Corresponding authors:}

Emmanouil Manolakos, Access to Genome - ATG Labs, 8 Sisini St., 11528 Athens, Greece manolakos@atg-labs.gr 00302107233323

Makarios Eleftheriades, Second Department of Obstetrics and Gynaecology, Aretaieion Hospital, Medical School, National and Kapodistrian University of Athens, 76 Vas. Sofias Avenue 11 528, Athens Greece.

makarios@hotmail.co.uk, 00306944223060 


\section{Established Facts and Novel Insights}

\section{Established Facts}

- The Microcephalin protein is a pleiotropic factor playing an important role in neurogenesis; it regulates the division of neuroprogenitor cells preventing them to exhaust.

- There are two categories of microcephaly: the first one is obvious during childbirth called primary microcephaly $(\mathrm{MCPH})$ while the second one developes later in life and is called secondary microcephaly.

\section{Novel Insights}

- Since the mutation c.348del has never been associated with microcephaly phenotypes, we reveal new findings of the disease spectrum linked to $M C P H 1$ gene mutations and its prenatal diagnosis.

- Using Next Generation Sequencing (NGS) analysis, the present study reveales a new homozygous mutation of $M C P H 1$ gene, thus expanding the disease spectrum of $M C P H 1$ gene.

\section{Abstract}

MCPH1, otherwise known as the microcephalin gene $\left({ }^{*} 607117\right)$ and protein, is a basic regulator of chromosome condensation (BCRT-BRCA1 C-terminus). The Microcephalin protein is made up of three BCRT domains and conserved tandem repeats of interacting phospho-peptide. There is a strong connection between mutations of the MCPH1 and reduced brain growth. Specifically, individuals with such mutations have underdeveloped brains which means smaller size, varying levels of mental retardation, delayed speech and poor language skills, individuals with mild microcephaly and normal intelligence notwithstanding. In this case, a fetus with novel homozygous mutation of the MCPH1 gene ((c.348del)), whose parents were recessive heterozygous for (c.348del), displayed severe microcephaly at 22 weeks of gestation. Due to the effect on splice sites in introns, this mutation causes forming of dysfunctional proteins which lack crucial domains of the C-terminus. Our findings portray an association between the new MCPH1 mutation ((c.348del)) and the clinical features of autosomal recessive primary microcephaly $(\mathrm{MCPH})$ contributing to a broader spectrum related to these pathologies. 
Keywords: microcephalin gene, homozygous, next generation sequencing, microcephaly. MCPH1 Abbreviations: NGS, next generation sequencing; $\mathrm{MCPH1}$, Microcephalin protein

Conflict of Interest: Nil

Source of support: None

\section{Introduction}

Autosomal recessive primary microcephaly $(\mathrm{MCPH})$ is an uncommon genetically heterogenous disorder of neurogenic brain development. Its characteristics are decreased head circumference during childbirth with no severe irregularities of the cerebrum and varying intellectual impairment. There are two categories of microcephaly: the first one is evident during childbirth and is called primary microcephaly $(\mathrm{MCPH})$ while the second one developes later in life and is called secondary microcephaly. The main difference between the two is that while MCPH is most of the times a static irregularity of development, secondary microcephaly is a dynamic neurodegenerative disorder. Patients with primary microcephaly have not severe mental issues and also do not show syndromic, neurologic or significant dysmorphias.

Whole genome (WGS) or whole exome (WES) studies identified 17 OMIM genes associated with MCPH and those are: WDR62 (*613583), CDK5RAP2 $\left({ }^{*} 608201\right)$, CASC5 $\left({ }^{*} 609173\right)$, ASPM $\left({ }^{*} 605481\right)$, CENPJ $\left({ }^{*} 609279\right)$, STIL $\left({ }^{*} 181590\right)$, CEP135 $\left({ }^{*} 611423\right)$, CEP152 $\left({ }^{*} 613529\right)$, ZNF335 $\left({ }^{*} 610827\right)$, PHC1 $\left({ }^{*} 602978\right)$, CDK6 $\left({ }^{*} 603368\right)$, CENPE $\left({ }^{*} 117143\right)$, CENPF $\left({ }^{*} 600235\right)$, PLK4 $\left({ }^{*} 605031\right)$, TUBGCP6 $\left({ }^{*} 610053\right)$, CEP63 $\left({ }^{*} 614724\right)$, NDE1 $\left({ }^{*} 609449\right)$ [1]. However, the first gene known to cause $\mathrm{MCPH}$ is $\left.\mathrm{MCPH} 1{ }^{*} 607117\right)$, making 18 genes in total associated with $\mathrm{MCPH}$ [2-3].

The location of MCPH1/microcephalin gene $\left({ }^{*} 607117\right)$ is chromosome $8 \mathrm{p} 23$. Cellular responses caused by DNA damage and chromosome condensation are dependent on microcephalin, the encoded protein [4]. This protein is involved in G2/M checkpoint arrest maintaining the inhibitory phosphorylation of cyclindependent kinase 1 . The ability to maintain normal brain size is lost when microcephalin is absent because it causes premature mitotic entry of neuroprogenitor cells [4-6].

In this study, we reported a case of a homozygous in MCPH1 gene (c.348del) fetus diagnosed at 22 weeks of gestation. The fetus was growth restricted showing microcephaly and partial agenesis of the corpus callosum. 


\section{Clinical Report}

A 30-year-old woman was referred to our center in her second pregnancy at 24 weeks of gestation, complicated with microcephaly $(\mathrm{GW})$, just as her first one had previously.

In the first pregnancy, during ultrasound examination (U/S) at 12 weeks of gestation the nuchal translucency measurement was within normal limits $(\mathrm{NT}=1.3 \mathrm{~mm})$ indicating low risk for Down syndrome (1/9052). The second trimester ultrasound examination (U/S) at 24 weeks of gestation revealed Fetal Growth Restriction (FGR), microcephaly and partial agenesis of the corpus callosum, dilatation of the third ventricle, right ureter dilatation and presence of a simple unilocular cyst at the lower lobe of the right kidney. After counselling the parents opted for fetal karyotyping and an amniocentesis was performed. Standard G-banding karyotyping revealed normal karyotype and chromosomal microarray analysis did not detect any pathogenic copy number variations. After genetic counselling the pregnancy was terminated at 26 weeks of gestation and an intact male fetus was delivered. The parents decided against autopsy and no further analysis was performed at this point. The parents were of Greek origin, healthy and nonconsanguineous.

In the second pregnancy, ultrasound examination (U/S) at 12 weeks of gestation revealed high risk for Down syndrome due to increased nuchal translucency measurement $(\mathrm{NT}=3.1 \mathrm{~mm})$. After counselling the parents opted for fetal karyotyping and chorionic villi sampling (CVS) was performed. Standard G-banding karyotyping revealed normal karyotype and chromosomal microarray analysis did not detect any pathogenic copy number variations. During the second trimester of pregnancy, ultrasound assessment showed severe microcephaly and FGR. Following pregnancy termination, fetal autopsy, standard G-banding karyotyping, chromosomal microarray and NGS analysis were performed.

\section{Material and Methods}

\section{Molecular karyotyping}

High-resolution molecular karyotyping was performed with an aCGH platform of 60,000 oligonucleotides (Agilent technologies). Briefly, DNA was extracted from the chorionic villus sample with Promega Maxwell 16, and it was hybridized with the human reference DNA of the same gender (Promega Biotech). The statistical test used as a parameter to estimate the number of copies was ADM-2 (provided by the DNA analytics software, Agilent Technologies) with a 
window of $0.5 \mathrm{Mb}$ and a threshold of 6 . Only those copy number changes that affected at least 5 consecutive probes with identically oriented change were considered as copy number variations. Consequently, for most of the genome, the average resolution of this analysis was $200 \mathrm{~kb}$.

\section{Next Generation Sequencing (NGS)}

DNA isolation from the fetus and the parents was performed. Following, Oligonucleotidebased target capture analysis and nucleotide sequencing was performed using TruSight One kit (Illumina) and Next Generation Sequencing (Illumina NextSeq), respectively, examining 99 disease-causing genes that were related with the clinical features of the fetus (Table 1). The software SOPHIA DDM $®$ (SOPHIA GENETICS) was used for the data analysis [reference genome UCSC hg19 and reference database Human Gene Mutation Database (HGMD v.2017.1)].

Table 1: 99 disease-causing genes that were related with the clinical features of the fetus.

\section{List of studied genes}

AP3B2, AP4B1, AP4E1, AP4M1, APEX1, ARFGEF2, ASNS, ASPM, ATR, ATRX, BMPRlA, BRWD3, C2, CDK5RAP2, CDON, CDT1, CENPJ, CEP152,COL2A1,CYP21A2,CZ1P-ASNS, DICER1, DPP6, EFTUD2, EHMT2, EMG1, F10, F2, F5, FAM20A, FAM20C, FLNA, FLT1, FN3K, GLI2, GNAS, HUWE1, HYMAI, IGF1R, KARS, KNL1, LIG4, LMNB2, LOC100287042, LOC102724058, MCM5, MCPH1, MCPH1-AS1, MED17, MMP2, MRE11, MTHFR, MTNR1A, MYH11, NBN, NCAPD2, NDE1, NELFE, NPC1, ORC4, ORC6, OSGEP, PACERR, PCNT, PEX2, PHB2, PLAGL1, PRKARIA, PRKDC, PTGS1, PTGS2, PTPRJ, REV3L, RPTOR, SCAP, SCARNA10, SCN10A, SCN1A, SERPINA3, SKIV2L, SLC16A2, SLC25A19, STIL, STK4, TBCD, TCOF1, TERT, TP53, TUBA1A, TUBGCP6, VPS13B, VPS35, VRK1, WDR62, WDR81, XRN1, ZNF335, ZNF592, ZNF750.

\section{Results}

Standard G-banding karyotyping and high-resolution molecular karyotyping revealed a normal chromosome chart of the second foetus. NGS analysis showed an inherited homozygosity for mutations of $\mathrm{MCPH1}$ gene (c.348del) which has not been reported in generalist polymorphism databases (ExaC or exome variant server (EVS)) (shown in Fig. 1). The results were confirmed by 
Sanger sequencing in this fetus and targeted sequencing on the parents showed that they were both heterozygous for the detected mutation (shown in Fig. 2). Following the diagnosis of the c.348del mutation of the second fetus, targeted sequencing of the DNA of the first fetus was performed showing homozygocity of the same (c.348del) mutation.

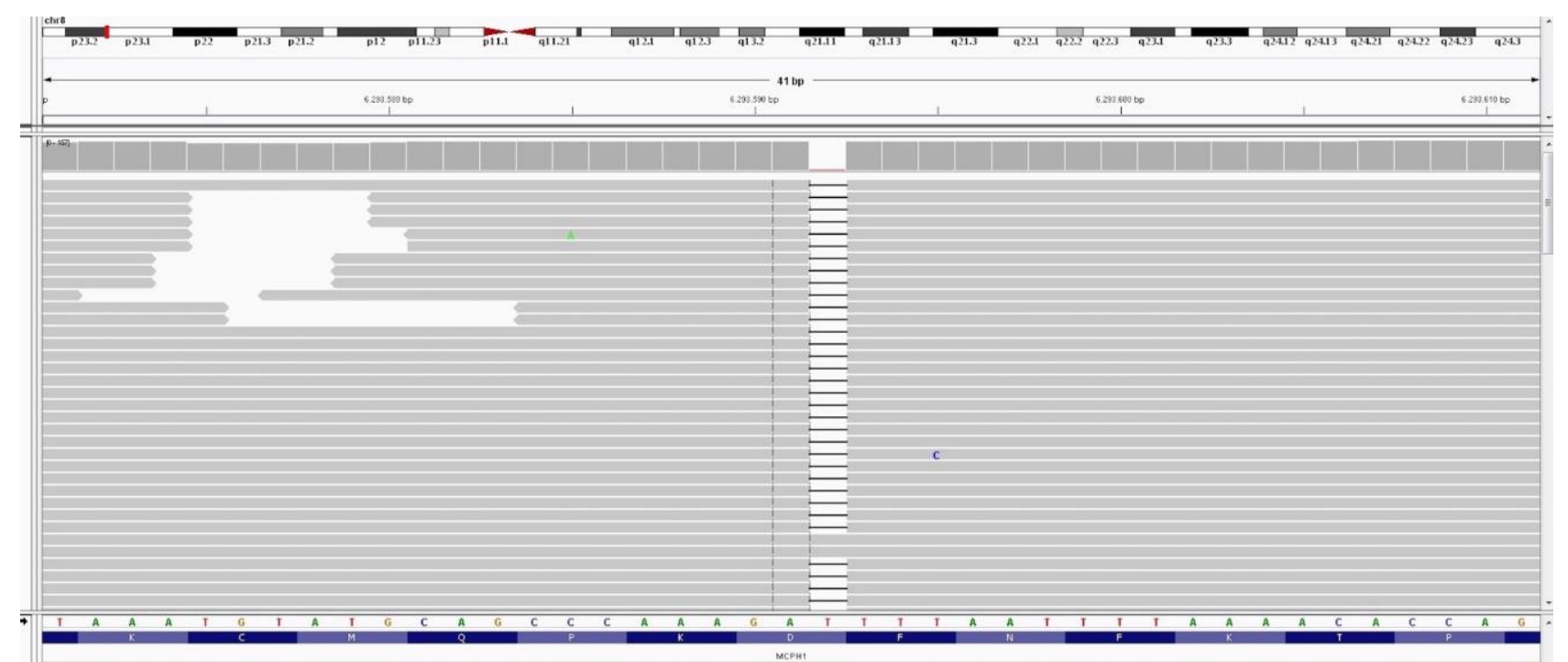

Figure 1: IGV screenshot for mutations in MCPH1 gene The mutation c.348del in MCPH1 gene in Homozygous state. (fetus 2)

A

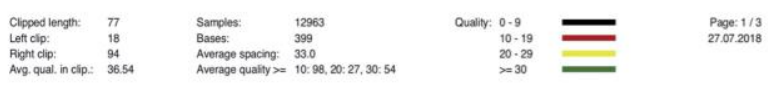

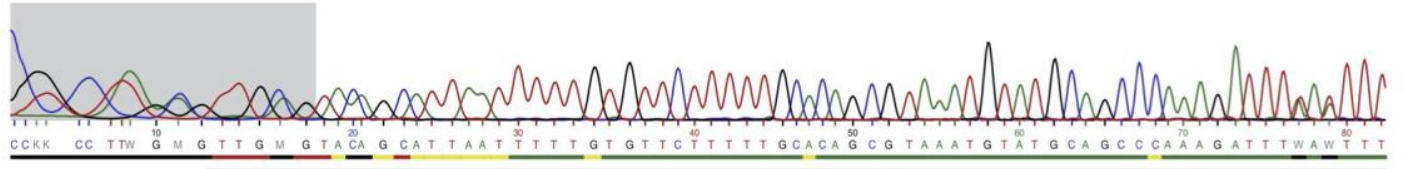

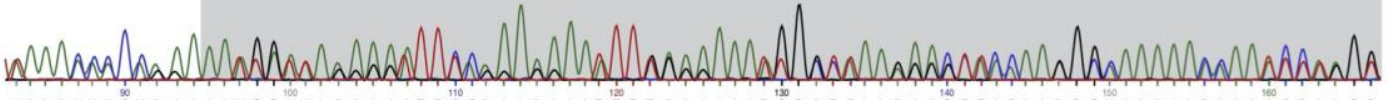



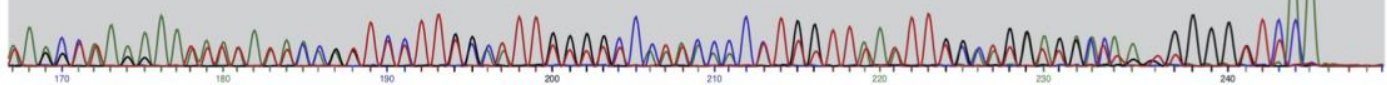



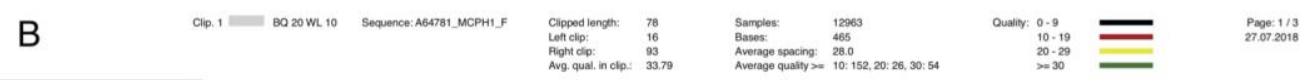

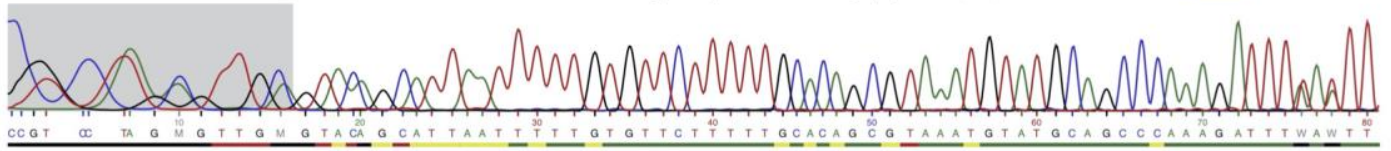

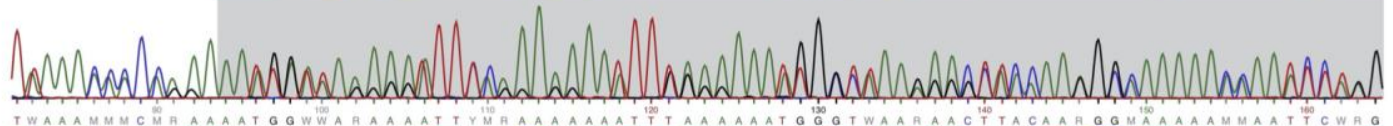

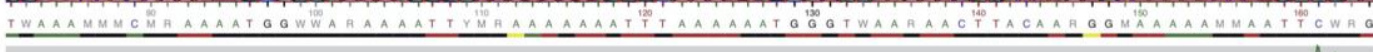

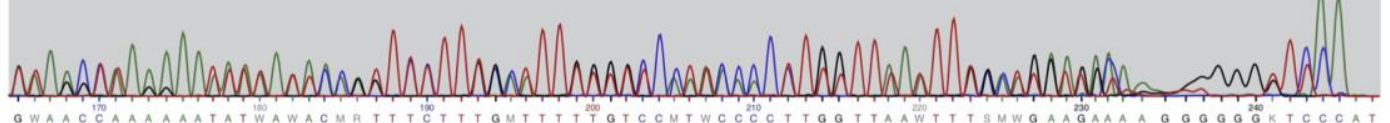

Figure 2.A: Sanger sequencing data of the mutation c.348del in MCPH1 gene in heterozygous state (mother). Figure 2.B:Sanger sequencing data of the mutation c.348del in MCPH1 gene in heterozygous state (father). 


\section{Histopathologic results}

Fetal necrotomy of fetus 2 included mild symmetric growth retardation, mild dysmorphic craniofacial features, microcephaly trend without true microencephaly, partial corpus callosum agenesis without histologic cerebral dysplasia. Placental weight was normal, but histologic examination revealed extended inflammatory lesions of maternal origin dating earlier in pregnancy (acute chorionitis and villitis, karyorrhexis, fibrin deposition), multifocal chronic villitis of unknown origin. There were no indications of a specific syndromic microcephaly and partial corpus callosum agenesis. The possibility of "microcephaly-corpus callosum agenesis syndrome" or other cases of isolated genetic microcephaly could not be excluded. The inflammatory lesions of the placenta could imply the possibility of intrauterine teratogenic effect due to an infectious agent, although no analogous histologic findings of the cerebrum were observed.

\section{Discussion}

The mode of inheritance of the genetically heterogenous disorder primary microcephaly $(\mathrm{MCPH})$ is autosomal recessive. $\mathrm{MCPH}$ causes dysmorphia of the brain with the characteristics of congenital small cranium with reduced occipito-frontal head diameter (OFD) of more than 2 standard deviations (SD) below the mean for ethnicity, age and sex (heavy microcephaly OFD $<3$ $\mathrm{SD}) \mathrm{MCPH}$ is further characterized by simplified gyral pattern, mild to moderate intellectual disability and mild seizures, polymicrogyria, periventricular neuronal heterotopias, hyperactivity and attention deficit disorder, speech delay, focal or generalized seizures, aggressiveness, and delay of developmental milestones and pyramidal signs [7-8].

The incidence of $\mathrm{MCPH}$ is about 1:30000-1:250000 live-births [9]. $\mathrm{MCPH}$ is less common in in whites than in Arab and Asian populations where consanguineous marriages are mutual [10]. More than 300 families worldwide have been reported to manifest MCPH.

241905bp is the genomic size of MCPH1/microcephalin gene. 835 aminoacids are encoded by 14 exons which are in the $8032 \mathrm{bp}$ open reading frame; until today, 3 isoforms are known [3]. The Microcephalin protein is a pleiotropic factor and plays an important role in neurogenesis; it regulates the division of neuroprogenitor cells preventing them to exhaust. It basically regulates the telomere integrity and associates with the DNA damage repair mechanism. The protein is involved in G2/M checkpoint arrest maintaining the inhibitory phosphorylation of cyclindependent kinase 1 [4-6]. It also operates as tumour suppressor in many human cancers, in germline functions and regulates the cerebral cortex size as well as the development of the brain 
[11-12]. Normal brain growth is dependent on MCPH1 gene that also acts as a positive selector for primate lineage [13].

Two types of mutations in MCPH1 have been described. Mild microcephaly and cellular phenotype are manifested in patients who were identified with a missense mutation [14-16]. Furthermore, there are reported deletions in MCPH1. An Iranian family with mild microcephaly and intellectual disability was identified with a deletion of the first 6 exons of the gene; at least $10 \%$ to $15 \%$ of cells had early chromosome condensation in this family [16]. Moreover, an Asian-Indian patient was identified with a deletion of the first 11 exons [17].

Hereditary hearing loss, facial dysmorphism and seizures as well as mild to severe mental retardation may be manifested in microcephaly patients [18]. There are two categories of microcephaly; the primary microcephaly when it is evident at birth and the secondary microcephaly when the disease is identified later in life [19]. Prenatal developmental neurogenic disorder relates to primary microcephaly while dynamic neurodegenerative disease relates to secondary microcephaly.

In this report, we present two consecutive pregnancies complicated by severe microcephaly caused by a homozygous mutation of the MCPH1 gene. Both parents of the embryo carried the recessive c.348del and were heterozygous. This mutation leads to the replacement of phenylalanine amino acid for leucine amino acid at position 116 of the protein altering the reading frame which cause an early stop codon at position 145 of the protein leading to protein forms which not function. The MCPH1 mutation (c.348del) has been revealed by our findings and appears to involve in microcephaly's clinical features contributing to MCPH1 spectrum's expansion associated to these pathologies. The mutations of $\mathrm{MCPH} 1$ gene were those which have been firstly identified in MCPH [8]. Furthermore, there have been reported few mutations in MCPH1 gene [18].

\section{Conclusion}

The diagnosis of primary microcephaly is based on the identification of MCPH1 mutations by molecular techniques. Although, we have found a novel mutation in homozygosity associated with fetal abnormalities, additional studies are necessary to verify the pathogenicity of this mutation and its definite association with fetal microcephaly. We present this case of the novel mutation c.348del to share information and expand our limited knowledge on the spectrum of disease linked to $\mathrm{MCPH1}$ gene mutations and its prenatal diagnosis. 


\section{Acknowledgment}

The authors would like to thank the family for their collaboration.

\section{Statement of Ethics}

The study was approved by the Ethics Committee of "Aretaieio" Hospital, Athens, Greece, and performed with respect to the ethical standards of the Declaration of Helsinki, as revised in 2008. The date of ethical approval was the $19^{\text {th }}$ of June, 2018 with code 60/19-06-18. Also, a written informed consent was obtained from the patient for publication of this case report and any accompanying images.

\section{Conflict of Interest Statement}

The authors declare no conflicts of interest. The datasets used and/or analyzed during the current study are available from the corresponding author on reasonable request.

\section{Funding Sources}

No funding sources to report.

\section{Author Contributions Statement}

All authors made substantive intellectual contributions, read and approved the final version of the manuscript. I. Papoulidis was in charge of overall patient management and prepared the initial draft of the manuscript. M. Eleftheriades, SM Liappi and Maria Papamichail were in preparation, and finalization of the manuscript providing important intellectual inputs. E. Manolakos, I. Papoulidis and SM Liappi performed and analyzed the WES data and the analysis of the identified variant. A. Konstantinidou participated in the histopathology assessment. M. Eleftheriades, V. Papadopoulos, A. Garas and S. Sotiriou interpreted clinical data and critically read the manuscript. I. Papastefanou and G. Daskalakis, performed ultrasound examination, cvs and amniocentesis respectively. A. Ristic supervised patient work-up and management. 


\section{References}

01. Morris-Rosendahl DJ, Kaindl AM. What next-generation sequencing (NGS) technology has enabled us to learn about primary autosomal recessive microcephaly (MCPH). Mol Cell Probes. 2015;29:271-81.

02. Jackson AP, McHale DP, Campbell DA, Jafri H, Rashid Y, Mannan J, Karbani G, Corry P, Levene MI, Mueller RF, Markham AF, Lench NJ, Woods CG. Primary autosomal recessive microcephaly (MCPH1) maps to chromosome 8p22-pter. Am J Hum Genet. 1998 Aug;63(2):541-6.

03. Jackson AP, Eastwood H, Bell SM, Adu J, Toomes C, Carr IM, Roberts E, Hampshire DJ, Crow YJ, Mighell AJ, Karbani G, Jafri H, Rashid Y, Mueller RF, Markham AF, Woods CG. Identification of microcephalin, a protein implicated in determining the size of the human brain. Am J Hum Genet. 2002 Jul;71(1):136-42.

04. Barbelanne M, Tsang WY. Molecular and cellular basis of autosomal recessive primary microcephaly. Biomed Res Int. 2014;2014:547986.

05. Cox J, Jackson AP, Bond J, Woods CG. What primary microcephaly can tell us about brain growth? Trends Mol Med. 2006;12:358-66.

06. Gruber R, Zho Z, Sukchev M, Joerss T, Frappart PO, Wang ZQ. MCPH1 regulates the neuroprogenitor division mode by coupling the centrosomal cycle with mitotic entry through the Chk1-Cdc25 pathway. Nat Cell Biol. 2011;13:1325-34.

07. Passemard S, Titomanlio L, Elmaleh M, Afenjar A, Alessandri JL, Andria G, Billette de Villemeur T, Boespflug-Tanguy O, Burglen L, Del Giudice E, Guimiot F, Hyon C, Isidor B, Mégarbané A, Moog U, Odent S, Hernandez K, Pouvreau N, Scala I, Schaer M, Gressens P, Gerard B, Verloes A. Expanding the clinical and neuroradiologic phenotype of primary microcephaly due to ASPM mutations. Neurology. 2009;73:962-9.

08. Kaindl AM, Passemard S, Kumar P, Kraemer N, Issa L, Zwirner A, Gerard B, Verloes A, Mani S, Gressens P. Many roads lead to primary autosomal recessive microcephaly. Prog Neurobiol. 2010 Mar;90(3):363-83.

09. Zaqout S, Morris-Rosendahl D \& Kaindl AM (2017). Autosomal recessiveprimary microcephaly (MCPH): an update. Neuropediatrics 48(3), 135-142.

10. Muhammad F, Mahmood Baig S, Hansen L, Sajid Hussain M, Anjum Inayat I, Aslam M, Anver Qureshi J, Toilat M, Kirst E, Wajid M \& Nürnberg P (2009). Compound heterozygous 
ASPM mutations in Pakistani MCPH families. American Journal of Medical Genetics Part A 149A(5), 926-930.

11. Venkatesh T, Nagashri MN, Swamy SS, Mohiyuddin SMA, Gopinath KS \& Kumar A (2013). Primary microcephaly gene MCPH1 shows signatures of tumor suppressors and is regulated by miR-27a in oral squamous cell carcinoma. PLOS One 8(3), e54643.

12. Pulvers JN, Journiac N, Arai Y \& Nardelli J (2015). MCPH1: a window into brain development and evolution. Frontiers in Cellular Neuroscience 9, 92.

13. Montgomery SH \& Mundy NI (2014). Microcephaly genes evolved adaptively throughout the evolution of eutherian mammals. BMC Evolutionary Biology 14(1), 120.

14. Woods CG, Bond J, Enard W. Autosomal recessive primary microcephaly (MCPH): a review of clinical, molecular, and evolutionary findings. Am J Hum Genet 2005; 76: 717-28.

15. Trimborn M, Bell SM, Felix C, Rashid Y, Jafri H, Griffiths PD, Neumann LM, Krebs A, Reis A, Sperling K, Neitzel H, Jackson AP. Mutations in microcephalin cause aberrant regulation of chromosome condensation. Am J Hum Genet. 2004;75:261-6.

16. Garshasbi M, Motazacker MM, Kahrizi K, Behjati F, Abedini SS, Nieh SE, Firouzabadi SG, Becker C, Rüschendorf F, Nürnberg P, Tzschach A, Vazifehmand R, Erdogan F, Ullmann R, Lenzner S, Kuss AW, Ropers HH, Najmabadi H. SNP array-based homozygosity mapping reveals $\mathrm{MCPH} 1$ deletion in family with autosomal recessive mental retardation and mild microcephaly. Hum Genet. 2006 Feb;118(6):708-15.

17. Pfau RB, Thrush DL, Hamelberg E, Bartholomew D, Botes S, Pastore M, Tan C, del Gaudio D, Gastier-Foster JM, Astbury C. MCPH1 deletion in a newborn with severe microcephaly and premature chromosome condensation. Eur J Med Genet. 2013 Nov;56(11):609-13.

18. Darvish H, Esmaeeli-Nieh S, Monajemi GB, Mohseni M, Ghasemi- Firouzabadi S, Abedini SS, Bahman I, Jamali P, Azimi S, Mojahedi F, Dehghan A, Shafeghati Y, Jankhah A, Falah M, Soltani , Banavandi MJ, Ghani M, Garshasbi M, Rakhshani F, Naghavi A, Tzschach A, Neitzel H, Ropers HH, Kuss AW, Behjati F, Kahrizi K \& Najmabadi H (2010). A clinical and molecular genetic study of 112 Iranian families with primary microcephaly. Journal of Medical Genetics $47(12), 823-828$.

19. Cowie V (1960). The genetics and sub-classification of microcephaly. Journal of Intellectual Disability Research 4(1), 42-47. 